\title{
Naver Labs Europe's Systems for the Document-Level Generation and Translation Task at WNGT 2019
}

\author{
Fahimeh Saleh* \\ Monash University \\ fahimeh.saleh@monash.edu
}

\author{
Alexandre Bérard \\ Naver Labs Europe \\ Ioan Calapodescu \\ first.last@naverlabs.com
}

\author{
Laurent Besacier \\ Université Grenoble-Alpes \\ laurent.besacier@univ-grenoble-alpes.fr
}

\begin{abstract}
Recently, neural models led to significant improvements in both machine translation (MT) and natural language generation tasks (NLG). However, generation of long descriptive summaries conditioned on structured data remains an open challenge. Likewise, MT that goes beyond sentence-level context is still an open issue (e.g., document-level MT or MT with metadata). To address these challenges, we propose to leverage data from both tasks and do transfer learning between MT, NLG, and MT with source-side metadata (MT+NLG). First, we train document-based MT systems with large amounts of parallel data. Then, we adapt these models to pure NLG and MT+NLG tasks by fine-tuning with smaller amounts of domain-specific data. This end-to-end NLG approach, without data selection and planning, outperforms the previous state of the art on the Rotowire NLG task. We participated to the "Document Generation and Translation" task at WNGT 2019, and ranked first in all tracks.
\end{abstract}

\section{Introduction}

Neural Machine Translation (NMT) and Neural Language Generation (NLG) are the top lines of the recent advances in Natural Language Processing. Although state-of-the-art NMT systems have reported impressive performance on several languages, there are still many challenges in this field especially when context is considered. Currently, the majority of NMT models translate sentences independently, without access to a larger context (e.g., other sentences from the same document or structured information). Additionally, despite improvements in text generation, generating long descriptive summaries conditioned on structured data is still an open challenge (e.g., table records). Ex-

${ }^{*}$ This work was done while the author was visiting at Naver Labs Europe. isting models lack accuracy, coherence, or adequacy to source material (Wiseman et al., 2017).

The two aspects which are mostly addressed in data-to-text generation techniques are identifying the most important information from input data, and verbalizing data as a coherent document: "What to talk about and how?" (Mei et al., 2016). These two challenges have been addressed separately as different modules in pipeline systems (McKeown, 1985; Reiter and Dale, 2000) or in an end-to-end manner with PCFGs or SMTlike approaches (Mooney and Wong, 2007; Angeli et al., 2010; Konstas and Lapata, 2013), or more recently, with neural generation models (Wiseman et al., 2017; Lebret et al., 2016; Mei et al., 2016). In spite of generating fluent text, end-to-end neural generation models perform weakly in terms of best content selection (Wiseman et al., 2017). Recently, Puduppully et al. (2019) trained an endto-end data-to-document generation model on the Rotowire dataset (English summaries of basketball games with structured data). ${ }^{1}$ They aimed to overcome the shortcomings of end-to-end neural NLG models by explicitly modeling content selection and planning in their architecture.

We suggest in this paper to leverage the data from both MT and NLG tasks with transfer learning. As both tasks have the same target (e.g., English-language stories), they can share the same decoder. The same encoder can also be used for NLG and MT if the NLG metadata is encoded as a text sequence. We first train domain-adapted document-level NMT models on large amounts of parallel data. Then we fine-tune these models on small amounts of NLG data, transitioning from MT to NLG. We show that separate data selection and ordering steps are not necessary if NLG model is trained at document level and is given

\footnotetext{
${ }^{1}$ https://github.com/harvardnlp/ boxscore-data
} 


\begin{tabular}{l|c|c|c|c} 
Corpus & Lang(s) & Split & Docs & Sents \\
\hline \multirow{2}{*}{ DGT } & & train & 242 & 3247 \\
& \multirow{2}{*}{ EN-DE } & valid & 240 & 3321 \\
& & test & 241 & 3248 \\
\hline \multirow{2}{*}{ Rotowire } & \multirow{2}{*}{ EN } & train & 3398 & $45.5 \mathrm{k}$ \\
& & valid & 727 & $9.9 \mathrm{k}$ \\
& & test & 728 & $10.0 \mathrm{k}$ \\
\hline WMT19-sent & \multirow{2}{*}{ EN-DE } & train & - & $28.5 \mathrm{M}$ \\
WMT19-doc & & & $68.4 \mathrm{k}$ & $3.63 \mathrm{M}$ \\
\hline \multirow{2}{*}{ News-crawl } & EN & train & $14.6 \mathrm{M}$ & $420 \mathrm{M}$ \\
& DE & & $25.1 \mathrm{M}$ & $534 \mathrm{M}$
\end{tabular}

Table 1: Statistics of the allowed resources. The English sides of DGT-train, valid and test are respectively subsets of Rotowire-train, valid and test. More monolingual data is available, but we only used Rotowire and News-crawl.

enough information. We propose a compact way to encode the data available in the original database, and enrich it with some extra facts that can be easily inferred with a minimal knowledge of the task. We also show that NLG models trained with this data capture document-level structure and can select and order information by themselves.

\section{Document-Level Generation and Translation Task}

The goal of the Document-Level Generation and Translation (DGT) task is to generate summaries of basketball games, in two languages (English and German), by using either structured data about the game, a game summary in the other language, or a combination of both. The task features 3 tracks, times 2 target languages (English or German): NLG (Data to Text), MT (Text to Text) and MT+NLG (Text + Data to Text). The data and evaluation are document-level, encouraging participants to generate full documents, rather than sentence-based outputs. Table 1 describes the allowed parallel and monolingual corpora.

\section{Our MT and NLG Approaches}

All our models (MT, NLG, MT+NLG) are based on Transformer Big (Vaswani et al., 2017). Details for each track are given in the following sections.

\subsection{Machine Translation Track}

For the MT track, we followed these steps:

1. Train sent-level MT models on all the WMT19 parallel data (doc and sent) plus DGT-train.

2. Back-translate (BT) the German and English News-crawl by sampling (Edunov et al., 2018).
3. Re-train sentence-level MT models on a concatenation of the WMT19 parallel data, DGTtrain and BT. The later was split into 20 parts, one part for each training epoch. This is almost equivalent to oversampling the non-BT data by 20 and doing a single epoch of training.

4. Fine-tune the best sentence-level checkpoint (according to valid perplexity) on documentlevel data. Like Junczys-Dowmunt (2019), we truncated the WMT documents into sequences of maximum 1100 BPE tokens. We also aggregated random sentences from WMT-sent into documents, and upsampled the DGT-train data. Contrary to Junczys-Dowmunt (2019), we do not use any sentence separator or document boundary tags.

5. Fine-tune the best doc-level checkpoint on DGT-train plus back-translated Rotowire-train and Rotowire-valid.

We describe the pre-processing and hyperparameters in Section 4. In steps (1) and (3), we train for at most 20 epochs, with early stopping based on newstest2014 perplexity. In step (4), we train for at most 5 additional epochs, with early stopping according to DGT-valid perplexity (doc-level). In the last step, we train for 100 epochs, with BLEU evaluation on DGT-valid every 10 epochs. We also compute the BLEU score of the best checkpoint according to DGT-valid perplexity, and keep the checkpoint with highest BLEU.

The models in step (5) overfit very quickly, reaching their best valid perplexity after only 1 or 2 epochs. For DE-EN, we found that the best DGTvalid BLEU was achieved anywhere between 10 and 100 epochs (sometimes with a high valid perplexity). For EN-DE, perplexity and BLEU correlated better, and the best checkpoint according to both scores was generally the same. The same observations apply when fine-tuning on NLG or $\mathrm{MT}+\mathrm{NLG}$ data in the next sections.

Like Berard et al. (2019), all our MT models use corpus tags: each source sentence starts with a special token which identifies the corpus it comes from (e.g., Paracrawl, Rotowire, News-crawl). At test time, we use the DGT tag.

One thing to note, is that document-level decoding is much slower than its sentence-level counterpart. $^{2}$ The goal of this document-level fine-tuning

\footnotetext{
${ }^{2}$ On a single V100, sent-level DGT-valid takes 1 minute to translate, while doc-level DGT-valid takes 6 minutes.
} 
was not to increase translation quality, but to allow us to use the same model for MT and NLG, which is easier to do at the document level.

\subsection{Natural Language Generation Track}

Original metadata consists of one JSON document per game, containing information about teams and their players. We first generate compact representations of this metadata as text sequences. Then, we fine-tune our doc-level MT models (from step 4) on the NLG task by using this representation on the source side and full stories on the target side. We train on a concatenation of DGT-train, Rotowire-train and Rotowire-valid. We filter the later to remove games that are also in DGT-valid. Our metadata has the following structure:

1. Date of the game as text.

2. Home team information (winner/loser tag, team name and city, points in the game, season wins and losses and teamlevel scores) and information about its next game (date, home/visitor tag, other team's name), inferred from the other JSON documents in Rotowire-train.

3. Visiting team information and details on its next game.

4. $N$ best players of the home team (player name, followed by all his non-zero scores in a fixed order and his starting position). Players are sorted by points first, then by rebounds and assists.

5. $N$ best players of the visiting team.

To help the models identify useful information, we use a combination of special tokens and positional information. For instance, the home team is always first, but a $<$ WINNER $>$ tag precedes the winning team and its players. We ignore all-zero statistics, but always use the same position for each type of score (e.g., points, then rebounds, then assists) and special tokens to help identify them (e.g., <PTS> 16 and $\angle$ REB $>8$ ). We try to limit the number of tags to keep the sequences short (e.g., made and attempted free throws and percentage: $\langle$ FT $>3$ 560 ). An example of metadata representation is shown in Table 2.

\subsection{MT+NLG Track}

For the MT+NLG track, we concatenate the MT source with the NLG data. We use the same metadata encoding method as in the NLG track and we fine-tune our doc-level MT models (from step 4). We also randomly mask tokens in the MT source (by replacing them with a $<$ MASK $>$ token), with $20 \%$ or $50 \%$ chance (with one different sampling per epoch). The goal is to force the model to use the metadata because of missing information in the source. At test time, we do not mask any token.

\section{Experiments}

\subsection{Data Pre-processing}

We filter the WMT19-sent parallel corpus with langid.py (Lui and Baldwin, 2012) and remove sentences of more than 175 tokens or with a length ratio greater than 1.5. Then, we apply the official DGT tokenizer (based on NLTK's word_tokenize) to the non-tokenized text (everything but DGT and Rotowire).

We apply BPE segmentation (Sennrich et al., 2016) with a joined SentencePiece-like model (Kudo and Richardson, 2018), with 32k merge operations, obtained on WMT + DGT-train (English + German). The vocabulary threshold is set to 100 and inline casing is applied (Berard et al., 2019). We employ the same joined BPE model and Fairseq dictionary for all models. The metadata is translated into the source language of the MT model used for initialization, ${ }^{3}$ and segmented into BPE (except for the special tokens) to allow transfer between MT and NLG. Then, we add a corpus tag to each source sequence, which specifies its origin (Rotowire, News-crawl, etc.)

Like Junczys-Dowmunt (2019), we split WMT19 documents that are too long into shorter documents (maximum 1100 BPE tokens). We also transform the sent-level WMT19 data into doclevel data by shuffling the corpus and grouping consecutive sentences into documents of random length. Finally, we upsample the doc-level data (WMT19 and DGT) by 8 times its original size (in terms of sent count). We do so by sampling random spans of consecutive sentences until reaching the desired size.

The DGT and Rotowire data is already tokenized and does not need filtering nor truncating. We segment it into BPE units and add corpus tags.

\subsection{Settings}

All the models are Transformer Big (Vaswani et al., 2017), implemented in Fairseq (Ott et al., 2018). We use the same hyper-parameters as Ott et al. (2018), with Adam and an inverse square root schedule with warmup (maximum LR 0.0005). We apply dropout and label smoothing with a rate of 0.1 . The source and target embeddings are shared and tied with the last layer. We train with halfprecision floats on $8 \mathrm{~V} 100$ GPUs, with at most 3500 tokens per batch and delayed updates of 10

\footnotetext{
${ }^{3}$ Only week days, months and player positions need to be translated.
} 


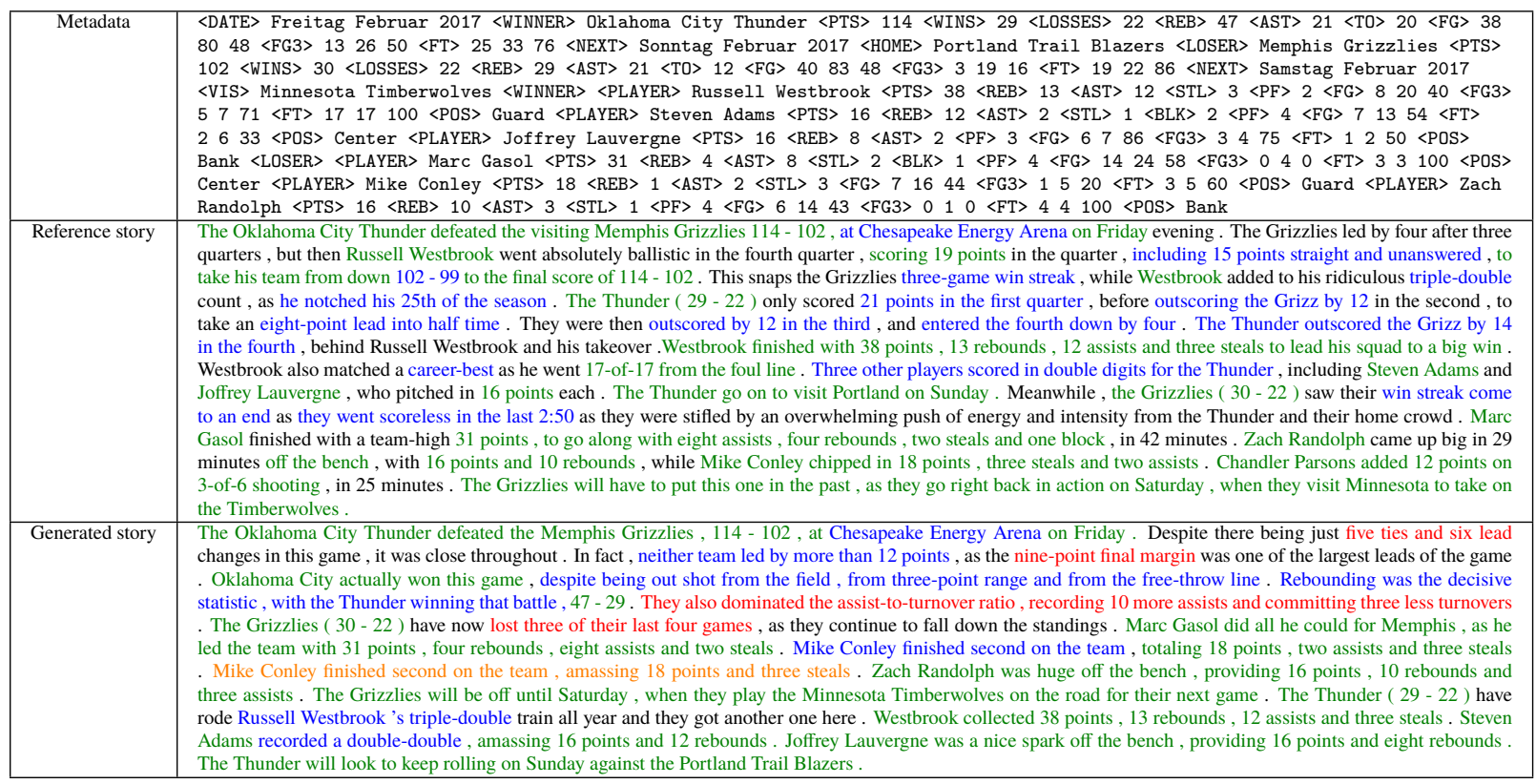

Table 2: Metadata: our metadata encoding. Reference story: story \#48 from DGT-valid. Generated story: output of the English NLG model (3-player). Green: text based on facts from the metadata. Blue: correct facts which are not explicitly in the metadata. Red: hallucinations or incorrect facts. Orange: repetitions.

\begin{tabular}{l|c|c|c|c} 
Track & Target & Constrained & Valid & Test \\
\hline NLG & & no & 23.5 & 20.5 \\
MT & \multirow{2}{*}{ EN } & yes & 60.2 & 58.2 \\
MT & & no & 64.2 & 62.2 \\
MT+NLG & & yes & 64.4 & 62.2 \\
\hline NLG & & no & 16.9 & 16.1 \\
MT & DE & yes & 49.8 & 48.0 \\
MT+NLG & & yes & 49.4 & 48.2
\end{tabular}

Table 3: Doc-level BLEU scores on the DGT valid and test sets of our submitted models in all tracks.

batches. When fine-tuning on DGT-train or Rotowire + DGT-train (Step 5 of the MT track, or NLG/MT+NLG fine-tuning), we use a fixed learning rate schedule (Adam with 0.00005 LR) and a much smaller batch size (1500 tokens on a single GPU without delayed updates). We train for 100 epochs, compute DGT-valid perplexity at each epoch, and DGT-valid BLEU every 10 epochs.

\subsection{BLEU evaluation}

Submitted models. For each track, we selected the best models according to their BLEU score on DGT-valid. The scores are shown in Table 3, and a description of the submitted models is given in Table 4. We compute BLEU using SacreBLEU with its tokenization set to none ${ }^{4}$ as the model outputs and references are already tokenized with NLTK. Hayashi et al. (2019) give the full results of

\footnotetext{
${ }^{4}$ SacreBLEU signature: BLEU+case.mixed+numrefs. $1+$ smooth.exp+tok.none+version.1.3.1
}

the task: the scores of the other participants, and values of other metrics (e.g., ROUGE). Our NLG models are "unconstrained" because the WMT19 parallel data, which we used for pre-training, was not allowed in this track. Similarly, we do two submissions for DE-EN MT: one constrained, where we fine-tuned the doc-level MT model on DGTtrain only, and one unconstrained, where we also used back-translated Rotowire-train and valid. All the MT and MT+NLG models are ensembles of 5 fine-tuning runs. Cascading the English NLG model with the ensemble of EN-DE MT models gives a BLEU score of 14.9 on DGT-test, slightly lower than the end-to-end German NLG model (16.1). We see that in the same data conditions (unconstrained mode), the MT+NLG models are not better than the pure MT models. Furthermore, we evaluated the MT+NLG models with MT-only source, and found only a slight decrease of $\approx 0.3$ BLEU, which confirms our suspicion that the NLG information is mostly ignored.

NMT analysis. Table 5 shows the BLEU scores of our MT models at different stages of training (sent-level, doc-level, fine-tuned), and compares them against one of the top contestants of the WMT19 news translation task (Ng et al., 2019).

English NLG analysis. Table 6 shows a 5.7 BLEU improvement on Rotowire-test by our English NLG model compared to the previous state 


\begin{tabular}{l|l|l} 
Track & N best players & Details \\
\hline NLG (EN) & 4 & Rotowire BT + DGT-train + tags \\
\hline NLG (DE) & 6 & Rotowire BT + DGT-train + tags \\
\hline MT (DE-EN) & N/A & $\begin{array}{l}\text { Unconstrained: } \text { Rotowire BT + } \\
\text { DGT-train + tags + ensemble } \\
\text { Constrained: DGT-train only + } \\
\text { ensemble }\end{array}$ \\
\hline MT (EN-DE) & N/A & DGT-train only + ensemble \\
\hline MT+NLG (EN) & 3 & $\begin{array}{l}\text { Rotowire BT + DGT-train + 20\% } \\
\text { text masking + tags + ensemble }\end{array}$ \\
\hline MT+NLG (DE) & 3 & $\begin{array}{l}\text { Rotowire BT + DGT-train + } \\
\text { tags + ensemble }\end{array}$
\end{tabular}

Table 4: Description of our submissions.

\begin{tabular}{c|c|c|c|c} 
Model & Target & Valid & Test & News 2019 \\
\hline FAIR 2019 & & 48.5 & 47.7 & $\mathbf{4 1 . 0}$ \\
Sent-level & \multirow{2}{*}{ EN } & 55.6 & 54.2 & 40.9 \\
Doc-level & & 56.5 & 55.0 & 38.5 \\
Fine-tuned & & $\mathbf{6 1 . 7}$ & $\mathbf{5 9 . 6}$ & 21.7 \\
\hline FAIR 2019 & & 37.5 & 37.0 & 40.8 \\
Sent-level & & 47.3 & 46.7 & $\mathbf{4 2 . 9}$ \\
Doc-level & DE & $\mathbf{4 8 . 2}$ & $\mathbf{4 7 . 5}$ & 41.6 \\
Fine-tuned & & 48.0 & 46.7 & 41.3
\end{tabular}

Table 5: BLEU scores of the MT models at different stages of training, and comparison with the state of the art. Scores on DGT-valid and DGT-test are doc-level, while News 2019 is sent-level (and so is decoding). On the latter, we used the DGT corpus tag for DE-EN, and the Paracrawl tag for EN-DE (we chose the tags with best BLEU on newstest2014). Scores by the "finetuned" models are averaged over 5 runs.

of the art. Figure 1 shows the DGT-valid BLEU scores of our English NLG models when varying the number of players selected in the metadata. We see that there is a sweet spot at 4 , but surprisingly, increasing the number of players up to 8 does not degrade BLEU significantly. We hypothesize that because the players are sorted from best to worst, the models learn to ignore the last players.

From Table 7, we see that sorting players helps, but only slightly. Using only team-level information, and no information about players gives worse but still decent BLEU scores.

Week day, player position or team-level aggregated scores can be removed without hurting BLEU. However, information about next games

\begin{tabular}{c|c} 
Model & Rotowire test \\
\hline Wiseman et al. (2017) & 14.5 \\
Puduppully et al. (2019) & 16.5 \\
Ours (4-player) & 22.2
\end{tabular}

Table 6: English NLG comparison against state-of-theart on Rotowire-test. BLEU of submitted NLG (EN) model, averaged over 3 runs. Because Rotowire tokenization is slightly different, we apply a set of fixes to the model outputs (e.g., 1-of-3 $\rightarrow 1$ - of - 3).

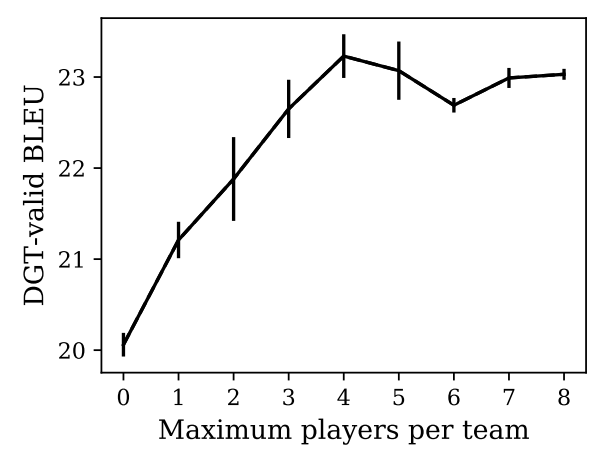

Figure 1: DGT-valid BLEU (by the best checkpoint) depending on the maximum number of selected players for the English NLG track.

\begin{tabular}{l|c|c} 
Model & Valid & Test \\
\hline Baseline (3 players, sorted) & $\mathbf{2 2 . 7}$ & 20.4 \\
\hline No player & 20.1 & 18.8 \\
All players, sorted & $\mathbf{2 2 . 7}$ & 20.9 \\
All players, shuffled & 22.0 & 20.0 \\
\hline (1) No next game & 22.0 & 19.9 \\
(2) No week day & 22.2 & 20.5 \\
(3) No player position & 22.6 & 20.5 \\
(4) No team-level sums & 22.5 & 20.5 \\
(5) Remove most tags & 22.6 & 20.8 \\
\hline (1) to (5) & 21.3 & 19.7
\end{tabular}

Table 7: English NLG ablation study, starting from a 3 best player baseline (the submitted NLG model has 4 players). BLEU averages over 3 runs. Standard deviation ranges between 0.1 and 0.4 .

seems useful. Interestingly, relying on position only and removing most tags (e.g., $\langle$ PTS $\rangle,\langle$ FT $\rangle$ ) seems to be fine. In this case, we also print all-zero stats, for the position of each statistic to be consistent across players and games.

Train-test overlap on Rotowire. We found a significant overlap between Rotowire train and test: 222 out of 728 Rotowire-test games are also in Rotowire-train (68/241 for DGT-test). The corresponding stories are always different but bear many similarities (some sentences are completely identical). Rotowire-train gets 24.2 BLEU when evaluated against Rotowire-test (subset of 222 stories). This gives us an estimate of human-level performance on this task. Our submitted NLG model gets 21.8 on the same subset. This overlap may cause an artificial increase in BLEU, that would unfairly favor overfitted models. Indeed, when filtering Rotowire-train to remove games that were also in DGT test, we found a slight decrease in BLEU (19.8 instead of 20.4). 


\begin{tabular}{|c|l|}
\hline Stadium name (+) & $\begin{array}{l}\text { REF: The Golden State Warriors ( 56 - 6 ) defeated the Orlando Magic ( 27 - 35 ) 119 - 113 } \\
\text { at Oracle Arena on Monday . } \\
\text { NLG: The Golden State Warriors ( } 56 \text { - 6 ) defeated the Orlando Magic ( 27 - 35 ) 119 - 113 } \\
\text { on Monday at Oracle Arena . }\end{array}$ \\
\hline Team alias (+) & $\begin{array}{l}\text { REF: The Heat held the Sixers to 38 percent shooting and blocked 14 shots in the win . } \\
\text { NLG: The Sixers shot just 38 percent from the field and 32 percent from the three-point line } \\
\text { while the Heat shot 44 percent from the floor and a meager 28 percent from deep . }\end{array}$ \\
\hline $\begin{array}{c}\text { Double-doubles or } \\
\text { triple-doubles (+) }\end{array}$ & $\begin{array}{l}\text { REF: Kevin Love 's 29-point , 13-rebound double-double led the way for the Cavs , who 'd } \\
\text { rested Kyrie Irving on Tuesday . } \\
\text { NLG: Love led the way for Cleveland with a 29-point , 13-rebound double-double that also } \\
\text { included three assists and two steals } .\end{array}$ \\
\hline Player injuries (-) & $\begin{array}{l}\text { NLG: The Timberwolves ( 28 - 44 ) checked in to Saturday 's contest with an injury-riddled } \\
\text { frontcourt, as Ricky Rubio ( knee ) and Karl-Anthony Towns ( ankle ) were sidelined . }\end{array}$ \\
\hline Ranking (-) & $\begin{array}{l}\text { NLG: The Heat ( 10 - 22 ) fell to 10 - 22 and remain in last place in the Eastern Conference 's } \\
\text { Southeast Division . }\end{array}$ \\
\hline $\begin{array}{c}\text { Season-level } \\
\text { player stats (-) }\end{array}$ & $\begin{array}{l}\text { NLG: It was a season-high in points for Thomas , who 's now averaging 17 points per game } \\
\text { on the season }\end{array}$ \\
\hline
\end{tabular}

Table 8: Correctly predicted information that is not explicitly in the metadata (+), or hallucinations (-).

\subsection{Qualitative evaluation}

As shown in Table 2, the NLG model (3player) has several good properties besides coherent document-level generation and the ability to "copy" metadata. It has learned generic information about the teams and players. As such, it can generate relevant information which is absent from metadata (see Table 8). For example, the model correctly predicts the name of the stadium where the game was played. This implies that it knows which team is hosting (this information is encoded implicitly by the position of the team in the data), and what is the stadium of this team's city (not in the metadata). Other facts that are absent from the metadata, and predicted correctly nonetheless, are team aliases (e.g., the Sixers) and player nicknames (e.g., the Greek Freak). The model can also generate other surface forms for the team names (e.g., the other Cavalier).

The NLG model can infer some information from the structured data, like double-digit scores, "double-doubles" (e.g., when a player has more than 10 points and 10 assists) and "triple-doubles". On the other hand, some numerical facts are inaccurate (e.g., score differences or comparisons). Some facts which are not present in the structured data, like player injuries, season-level player statistics, current ranking of a team, or timing information are hallucinated. We believe that most of these hallucinations could be avoided by adding the missing facts to the structured data. More rarely, model duplicates a piece of information.

Another of its flaws is a poor generalization to new names (team, city or player). This can quickly be observed by replacing a team name by a fictional one in the metadata. In this case, the model almost always reverts to an existing team. This may be due to overfitting, as earlier checkpoints seem to handle unknown team names better, even though they give lower BLEU. This generalization property could be assessed by doing a new train/test split, that does not share the same teams.

\section{Conclusion}

We participated in the 3 tracks of the DGT task: MT, NLG and MT+NLG. Our systems rely heavily on transfer learning, from document-level MT (high-resource task) to document-level NLG (lowresource task). Our submitted systems ranked first in all tracks.

For the MT task, the usual domain adaptation techniques performed well. The MT+NLG models did not show any significant improvement over pure MT. The MT models are already very good and probably do not need the extra context (which is generally encoded in the source-language summary already). Finally, our NLG models, bootstrapped from the MT models, do fluent and coherent text generation and are even able to infer some facts that are not explicitly encoded in the structured data. Some of their current limitations (mostly hallucinations) could be solved by adding extra information (e.g., injured players, current team rank, number of consecutive wins, etc.)

Our aggressive fine-tuning allowed us to specialize MT models into NLG models, but it will be interesting to study whether a single model can solve both tasks at once (i.e., with multi-task learning), possibly in both languages. 


\section{References}

Gabor Angeli, Percy Liang, and Dan Klein. 2010. A Simple Domain-Independent Probabilistic Approach to Generation. In EMNLP.

Alexandre Berard, Calapodescu Ioan, and Claude Roux. 2019. NAVER LABS Europe's Systems for the WMT19 Machine Translation Robustness Task. In WMT - Shared Task Paper.

Sergey Edunov, Myle Ott, Michael Auli, and David Grangier. 2018. Understanding Back-Translation at Scale. In EMNLP.

Hiroaki Hayashi, Yusuke Oda, Alexandra Birch, Ioannis Constas, Andrew Finch, Minh-Thang Luong, Graham Neubig, and Katsuhito Sudoh. 2019. Findings of the Third Workshop on Neural Generation and Translation. In Proceedings of the Third Workshop on Neural Generation and Translation (WNGT).

Marcin Junczys-Dowmunt. 2019. Microsoft Translator at WMT 2019: Towards Large-Scale DocumentLevel Neural Machine Translation. In WMT - Shared Task Papers.

Ioannis Konstas and Mirella Lapata. 2013. A Global Model for Concept-to-Text Generation.

Taku Kudo and John Richardson. 2018. SentencePiece: A simple and language independent subword tokenizer and detokenizer for Neural Text Processing. In EMNLP.

Rémi Lebret, David Grangier, and Michael Auli. 2016. Neural Text Generation from Structured Data with Application to the Biography Domain. In EMNLP.

Marco Lui and Timothy Baldwin. 2012. Langid.Py: An Off-the-shelf Language Identification Tool. In Proceedings of the ACL 2012 System Demonstrations, ACL.

Kathleen R. McKeown. 1985. Text Generation: Using Discourse Strategies and Focus Constraints to Generate Natural Language Text. Cambridge University Press.

Hongyuan Mei, Mohit Bansal, and Matthew R Walter. 2016. What to talk about and how? Selective Generation using LSTMs with Coarse-to-Fine Alignment. In NAACL-HLT.

Raymond J Mooney and Yuk Wah Wong. 2007. Generation by Inverting a Semantic Parser that Uses Statistical Machine Translation. In NAACL-HLT.

Nathan Ng, Kyra Yee, Alexei Baevski, Myle Ott, Michael Auli, and Sergey Edunov. 2019. Facebook FAIR's WMT19 News Translation Task Submission. In WMT - Shared Task Papers.

Myle Ott, Sergey Edunov, David Grangier, and Michael Auli. 2018. Scaling Neural Machine Translation. In WMT.
Ratish Puduppully, Li Dong, and Mirella Lapata. 2019. Data-to-Text Generation with Content Selection and Planning. In Proceedings of the AAAI Conference on Artificial Intelligence.

Ehud Reiter and Robert Dale. 2000. Building Natural Language Generation Systems. Cambridge University Press.

Rico Sennrich, Barry Haddow, and Alexandra Birch. 2016. Neural Machine Translation of Rare Words with Subword Units. In $A C L$.

Ashish Vaswani, Noam Shazeer, Niki Parmar, Jakob Uszkoreit, Llion Jones, Aidan N. Gomez, Lukasz Kaiser, and Illia Polosukhin. 2017. Attention Is All You Need. In NIPS.

Sam Wiseman, Stuart Shieber, and Alexander Rush. 2017. Challenges in Data-to-Document Generation. In EMNLP. 